\title{
Focus distance-aware lifetime maximization of video camera-based wireless sensor networks
}

\author{
André Rossi ${ }^{1}\left[\right.$ [1] Alok Singh ${ }^{2} \cdot$ Marc Sevaux $^{3}$ \\ Received: 1 October 2018 / Revised: 29 March 2019 / Accepted: 21 September 2019 \\ (c) Springer Science+Business Media, LLC, part of Springer Nature 2019
}

\begin{abstract}
The problem of maximizing the lifetime of a wireless sensor network which uses video cameras to monitor targets is considered. These video cameras can rotate and have a fixed monitoring angle. For a target to be covered by a video camera mounted on a sensor node, three conditions must be satisfied. First, the distance between the sensor and the target should be less than the sensing range. Second, the direction of the camera sensor should face the target, and third, the focus of the video camera should be such that the picture of the target is sharp. Basic elements on optics are recalled, then some properties are shown to efficiently address the problem of setting the direction and focal distance of a video camera for target coverage. Then, a column generation algorithm based on these properties is proposed for solving three lifetime maximization problems. Targets are considered as points in the first problem, they are considered as discs in the second problem (which allows for considering occlusion) and in the last problem, focal distance is also dealt with for taking image sharpness into account. All of these problems are compared on a testbed of 180 instances and numerical results show the effectiveness of the proposed approach.
\end{abstract}

Keywords Wireless sensor networks · Camera sensors · Column generation · Genetic algorithms

André Rossi

andre.rossi@dauphine.psl.eu

Alok Singh

alokcs@uohyd.ernet.in

Marc Sevaux

marc.sevaux@univ-ubs.fr

1 Université Paris-Dauphine, PSL Research University, CNRS, UMR 7243, LAMSADE, Paris, France

2 School of Computer and Information Sciences, University of Hyderabad, Hyderabad, India

3 Université de Bretagne-Sud, Lab-STICC, CNRS UMR 6285, Lorient, France 


\section{Introduction}

The spectacular progress in wireless communication and embedded systems technologies have made possible the availability of small and cheap wireless camera sensor networks (Yick et al. 2008; Queiroz et al. 2017), thereby paving the way for new monitoring applications (Liu 2014). This is particularly relevant in contexts where power is not available, as for outdoor agriculture (Garcia-Sanchez et al. 2011) and barrier crossing detection (Tao et al. 2012). The wireless sensors that are considered are then powered by a non-rechargeable battery, and the problem's objective is to use them in order to cover all the targets for the maximum amount of time (it is a lifetime maximization problem). As a first approximation, a camera sensor can be considered as a directional sensor. This is convenient since there exists a vast literature on directional sensor networks and many authors have used directional sensors to model camera sensors (Guo et al. 2016; Wang et al. 2018). Camera sensors are used to monitor regions, and scheduling is computed dynamically as a way to put more focus on those critical regions where intrusions, for example, have recently been observed (Jianjian et al. 2018; Han et al. 2019). Alternatively, Wang et al. (2009a) proposes a genetic algorithm (GA) to address a target priority-based version of the problem. Other works consider target tracking by taking advantage of the rotation of video cameras, but without taking image sharpness into account (De et al. 2012; Yang et al. 2015). Mobility and motility are combined in Guvensan and Yavuz (2013) to improve coverage with directional sensors, distributed approaches based on Voronoi diagrams (Sung and Yang 2014) and Lagrangian relaxation (Astorino et al. 2018) have also been proposed. Coverage with mobile directional sensors is addressed in Li et al. (2016), and distributed approaches for minimizing the number of active sensors and coverage overlapping are introduced in Sharmin et al. (2017).

Although camera sensors have received a lot of attention recently (Hong et al. 2017; Ji et al. 2017; Li et al. 2015), they are still modeled as directional sensors, without considering that a picture has to be reasonably sharp for being exploitable. Optics laws make it impossible to ensure that all the objects in a picture are sharp, in particular when some of them are very close to the camera, and some others are very far away from it Guvensan and Yavuz (2011). Because of the limited depth of field of the camera, the focal distance of the camera lens should be adjusted in order to select a set of objects to be sharp on the picture. This implies that in addition to the camera direction (that is taken into account by the directional sensor model), an additional decision has to be made on the lens focal distance in order to select the objects that will be sharp on the picture.

This paper is the first one in which the problem of maximizing the lifetime of a wireless camera sensor network for target coverage by optimally selecting the activation time, the direction, and the focal distance of each camera, is addressed. In order to evaluate the practical impact of the last parameter (focal distance), we also consider the size of the targets. This means that additional phenomena, like occlusion and image sharpness, have to be managed. Then, the first directional sensor model where all the targets are punctual and supposed to be sharp (referred to as 'd') is compared with the two new proposed camera sensor models. The second model considers occlusion, i.e., targets are discs of a nonzero diameter, but not focal distance, which means that image 
sharpness is not taken into account; this model is denoted by ' $\mathrm{g}$ ', for geometry. Finally, the third model, (' $\mathrm{f}$ ' for full) is the more realistic one, and both considers occlusion and sharpness. Those three problem variants are addressed using an exact approach based on column generation hybridized with a GA. Thus, the impact of occlusion can be evaluated by comparing models ' $\mathrm{d}$ ' and ' $\mathrm{g}$ ', and the impact of focal distance can be highlighted by comparing models ' $g$ ' and 'f'. Since the first problem is a particular case of the second one, which is itself a particular case of the third one, the proposed model is given for the third problem.

The present paper is an extension of the work presented in Rossi et al. (2013) for directional sensor networks to camera sensor networks and brings the following contributions. First, the problem addressed in Rossi et al. (2013) is extended to more realistic variants with the introduction of the focal distance as a new parameter, and also by taking occlusion into account. Theoretical properties and algorithm are proposed, as in Rossi et al. (2012b), in order to produce redundancy-free mathematical models. Upper bounds are introduced for assessing the quality of the current solution during the search. Finally, an analysis of the numerical results on the three variants shows that addressing more realistic versions of the problem is beneficial to the computational time required. We assume that sensors are randomly deployed, so we do not consider sensor placement in this work. The readers interested in camera sensor placement are referred to Lin et al. (2011).

The remainder of this paper is organized as follows. Section 2 introduces the optics background used in this paper and states the conditions under which a target is said to be covered by a sensor. Section 3 provides some theoretical properties, and introduces the algorithms for generating the necessary data required by the solution approach. A column generation algorithm is proposed in Sect. 4, with upper bounds on the network lifetime as well as a GA for generating efficient columns. Section 5 provides numerical experiments and discusses the results, and Sect. 6 concludes the paper.

\section{Target coverage using optical video camera}

\subsection{Optical characteristics of camera sensors}

We use the same parameter settings as in Guvensan and Yavuz (2011). A video camera is characterized by its focal length $f=50 \mathrm{~mm}$, the f-number $N=\frac{f}{d}=2.8$ that is the diameter of the entrance pupil in terms of the focal length, where $d$ is the effective aperture diameter, in $\mathrm{mm}$, and the circle of confusion $c=11 \mathrm{~mm}$ which control the acceptable sharpness of pictures. We arbitrary set $R$, the sensing range, to 150 meters, saying that if a target is farther than $R$ from the sensor, the picture quality and resolution is not considered good enough for identifying a target. The monitoring angle $\varphi \in(0,2 \pi]$ is a given parameter of a sensor that defines the maximum angular magnitude covered by the sensor. An omni-directional sensor has a monitoring angle of $2 \pi$, whereas an optical sensor like a camera has a monitoring angle that cannot exceed $\pi$. 


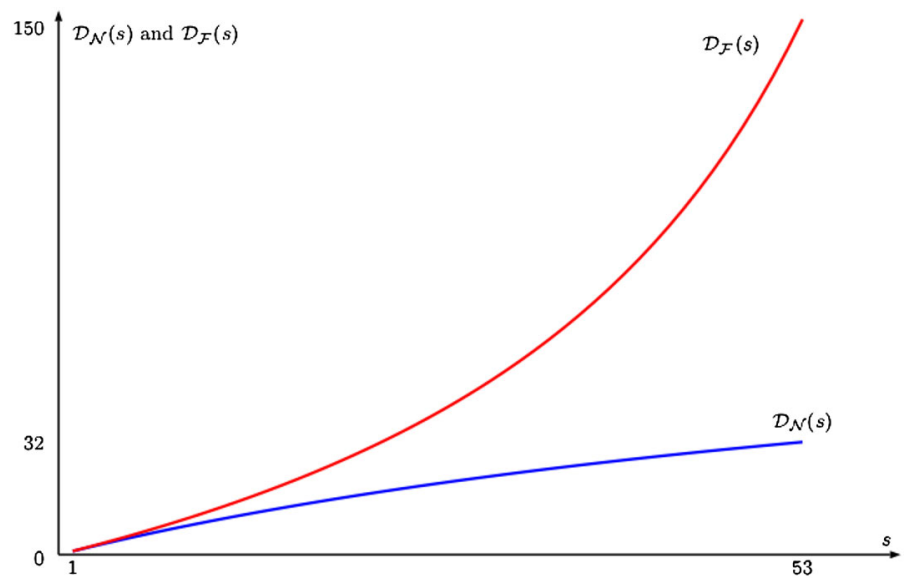

Fig. 1 Near and far distances of acceptable sharpness as a function of the focal distance $s$

We use the following formulas (taken from Guvensan and Yavuz 2011) for defining the hyper-focal distance $H$ and the depth of field of a camera sensor as a function of the focal distance $s$ :

$-H=\frac{f^{2}}{N c}+f$ is the hyper-focal distance, that is the maximum value for $s$ : if $s=H$, then all the objects from $s$ to infinity are sharp.

$-\mathcal{D}_{\mathcal{N}}(s)=\frac{s(H-f)}{H+s-2 f}$ is the near distance of acceptable sharpness in meters.

$-\mathcal{D}_{\mathcal{F}}(s)=\frac{s(H-f)}{H-s}$ is the far distance of acceptable sharpness in meters.

A graphical representation of $\mathcal{D}_{\mathcal{N}}(s)$ and $\mathcal{D}_{\mathcal{F}}(s)$ as a function of $s$ is shown in Fig. 1. The depth of field of the camera for a given $s$ is $\mathcal{D}_{\mathcal{F}}(s)-\mathcal{D}_{\mathcal{N}}(s)$. As can be seen on the figure, it is increasing with $s$.

The distance between the centers of sensor $i$ and target $k$ is denoted by $d_{i s t_{i, k}}$ for all $(i, k) \in\{1, \ldots, n\} \times\{1, \ldots, m\}$. Let $D_{i}$ be the set of all the targets whose distance to $i$ is less than or equal to $R$. If targets are discs of radius $r_{t}$,

$$
D_{i}=\left\{k \in\{1, \ldots, m\}: \text { dist }_{i, k}+r_{t} \leq R\right\} \quad \forall i \in\{1, \ldots, n\}
$$

The maximum focal distance $s_{\max }$ can be deduced from the relation $\mathcal{D}_{\mathcal{F}}\left(s_{\text {max }}\right)=$ $R$ :

$$
s_{\text {max }}=\frac{H R}{H-f+R}
$$

Similarly, we can deduce that $s_{\text {min }}=f$ by observing that $\mathcal{D}_{\mathcal{N}}\left(s_{\text {min }}\right)=\mathcal{D}_{\mathcal{F}}\left(s_{\text {min }}\right)$. Consequently, $s$ is in the interval $\left[s_{\min }, s_{\max }\right]$. 

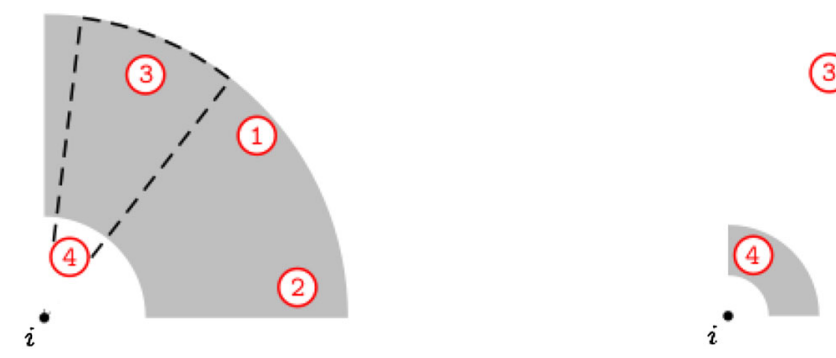

Fig. 2 Illustrating occlusion and focal distance for the same working direction

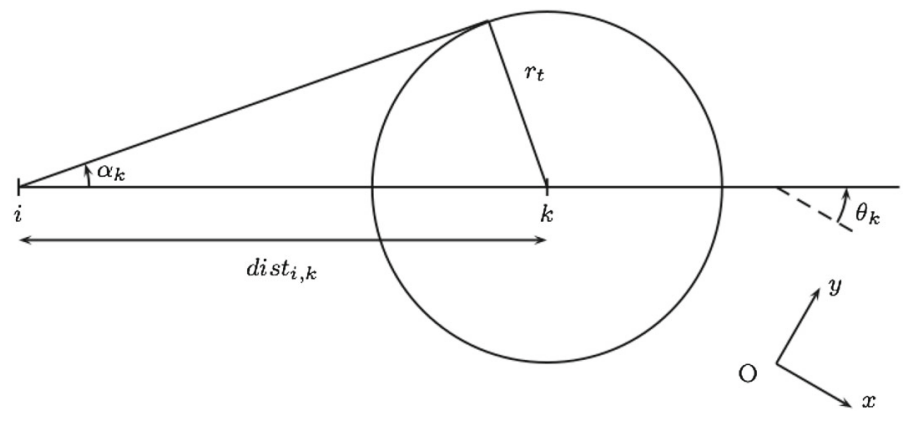

Fig. 3 Computation of the angle under which target $k$ is seen from sensor $i$

\subsection{Occlusion}

Occlusion occurs when a target hides a more distant one from a sensor. As an example, target 3 is occluded by target 4 in Fig. 2 .

Let $\theta_{k}$ be the angle under which the center of target $k$ is seen from sensor $i$ in the reference frame $\mathrm{O} x y$. Target $k$ is seen from $i$ under angle $2 \alpha_{k}$, where $\alpha_{k}$ is the angle between the line joining $i$ to $k$, and the tangent to the circle of center $k$ and radius $r_{t}$, passing through $i$. This angle is shown in Fig. 3, where it can be seen that $\alpha_{k}=\arcsin \left(\frac{r_{t}}{d i s t_{i, k}}\right)$. Furthermore $\alpha_{k} \in[0, \pi)$, and the whole target is seen from $i$ under the angular interval $I_{i, k}=\left[\theta_{k}-\alpha_{k}, \theta_{k}+\alpha_{k}\right]$.

Occlusion is detected as follows. Let $k$ and $k^{\prime}$ be two targets such that dist $_{i, k} \leq$ $d i s t_{i, k^{\prime}} \leq R$. We say that $k$ occludes $k^{\prime}$ from $i$ if the two angular intervals $I_{i, k}$ and $I_{i, k^{\prime}}$ overlap. Hence, because of occlusion, a target may not be covered by a neighboring sensor because of another target in between itself and the considered sensor. Consequently, the list of all the targets that can be covered by sensor $i$ can be found by considering all the pairs $\left(k, k^{\prime}\right) \in D_{i}$ with $k \leq k^{\prime}$. The whole process then requires at most $\mathcal{O}(\mathrm{nm})$ operations.

A less strict definition of occlusion (i.e., where partial occlusion might be considered acceptable) could have been adopted; additionally we could have considered targets having a non-circular shape (or different radii). Alternative choices regarding these two matters would have lead to a different computation of $\alpha_{k}$ for accounting for targets 
geometry, and to an algorithm where the actual overlapping would be computed and taken into account for deciding if a target is considered visible enough for being covered by a sensor. However, these choices are limited to the definition of the groups, and have no impact on the proposed approach to optimize the network lifetime.

\subsection{Target coverage}

The problem of maximizing lifetime is to build a set of groups. Each group is a subset of active sensors, and for each active sensor, we must determine a direction $\theta$ (i.e., an angle) in $[0,2 \pi)$ and a focal distance $s$ (in meters). A group is said to be valid if all the targets are covered by at least one sensor in the group. Groups are indexed in $\{1, \ldots, p\}$, and group $j$ is used for $t_{j}$ units of time. All sensors that do not belong to the group are in sleep mode, and do not consume energy. The locus of the points that can be sharply seen from a sensor is an annular sector, shown as a shaded region in Fig. 2. As a result, every target $k$ that satisfy the three following conditions is said to be covered by sensor $i$ under its direction $\theta$ with focal distance $s \in\left[s_{\min }, s_{\max }\right]$ :

- The image of target $k$ captured by sensor $i$ is sharp, which can be expressed as

$$
\left\{\begin{array}{l}
\text { dist }_{i, k}-r_{t} \geq \mathcal{D}_{\mathcal{N}}(s) \\
\text { dist }_{i, k}+r_{t} \leq \mathcal{D}_{\mathcal{F}}(s)
\end{array}\right.
$$

These constraints state that the closest point of the target (respectively the farthest one) which is at distance $d i s t_{i, k}-r_{t}$ (respectively at distance $d i s t_{i, k}+r_{t}$ ) from $i$, has to be sharp.

- The angle $\theta_{k}$ under which target $k$ is seen from sensor $i$ satisfies

$$
\begin{aligned}
& \left(\theta+\alpha_{k}\right) \bmod 2 \pi \leq \theta_{k} \leq\left(\theta+\varphi-\alpha_{k}\right) \bmod 2 \pi \\
& \text { if }\left(\theta+\alpha_{k}\right) \bmod 2 \pi \leq\left(\theta+\varphi-\alpha_{k}\right) \bmod 2 \pi \\
& \left(\theta+\alpha_{k}\right) \bmod 2 \pi \leq \theta_{k} \text { or } \theta_{k} \leq\left(\theta+\varphi-\alpha_{k}\right) \bmod 2 \pi \text { otherwise }
\end{aligned}
$$

These constraints enforce that target $k$ is fully seen from $i$ despite the limited monitoring angle of the sensor (mod stands for the arithmetic modulo operator). This is achieved by ensuring that the angular sector under which $k$ is seen from $i$ is included into the angular interval that is visible when the direction of $i$ is $\theta$. The first constraint covers the case where the angular sector under which $k$ is seen from $i$ is an interval of the form $[a, b]$ whereas the second constraint is for the case where this angular sector is not an interval, and is of the form $[0, a] \cup[b, 2 \pi)$, which may arise because the angles are in the interval $[0,2 \pi)$.

- Target $k$ is not occluded by another target.

Indeed, if a target is completely or partially hidden by another target, then it is not considered visible by the sensor.

For example, on Fig. 2, left, sensor $i$ has a monitoring angle of $\frac{\pi}{2}$ radians, and it cannot cover target 3 because it is occluded by target 4 . The occluded zone is shown in dashed line. Moreover, target 4 is too close from sensor $i$ for being covered together 
with targets 1 and 2. On Fig. 2, right, target 4 is covered. The sensor direction is the same in both the sub-figures of Fig. 2, but the focal distance is different, hence the covered areas (and targets) differ. However, we assume that power consumption is the same in both cases.

\section{Data preprocessing}

\subsection{Dominance properties}

The problem of Lifetime Maximization with Directional Sensors and Focal Distance is denoted by LM-DSFD. It is $\mathcal{N} \mathcal{P}$-hard as it is an extension of LM-CDS (Lifetime Maximization with Directional Sensors) which has been proven $\mathcal{N} \mathcal{P}$-hard in Rossi et al. (2013). Indeed, LM-CDS is a particular case of LM-DSFD where the focal distance is fixed.

A solution to LM-DSFD is a collection of valid groups, such that the sum of the duration of these groups is maximized, while ensuring that the battery limitation of each sensor is not exceeded. It follows that group $j$ is associated the following decision variables: $t_{j}$, and an annular sector $(\theta, s)$ for each sensor used in group $j$. For each sensor, $Z_{\theta, s}$ is the set of all the targets that are covered by this sensor when its direction is $\theta$ and its focal distance is $s$. Since $\theta$ and $s$ are physical continuous variables, the number of distinct annular sectors $(\theta, s)$ for a sensor is infinite. However, a finite number of annular sectors can be considered for target coverage: for each sensor, the $(\theta, s)$ annular sector is said to be normalized if the following two conditions are met:

1. There exists a target $k \in Z_{\theta, s}$ such that $\theta_{k}=\left(\theta+\alpha_{k}\right) \bmod 2 \pi$

2. There exists a target $k^{\prime} \in Z_{\theta, s}$ such that $\mathcal{D}_{\mathcal{F}}(s)=$ dist $_{i, k^{\prime}}+r_{t}$

We may have $k=k^{\prime}$ in the aforementioned conditions; this happens in particular when an annular sector covers a single target. A normalized group is a group for which all active sensors are set up to a normalized annular sector $(\theta, s)$.

Lemma 1 Any solution to LM-DSFD can be transformed into a solution where normalized annular sectors only are used, with the same lifetime.

Without loss of generality, we assume that any annular sector covers at least one target (if $Z_{\theta, s}$ is empty, the corresponding sensor can be set inactive in the considered group). If a solution to LM-DSFD contains a non-normalized annular sector $(\theta, s)$ with some sensor $i$ in some group $j$, then this annular sector can be replaced by a normalized annular sector of the same sensor by performing the following two steps:

1. $\theta$ is set to $\Theta_{k}=\left(\theta_{k}-\alpha_{k}\right) \bmod 2 \pi$, where target $k \in Z_{\theta, s}$ requires the minimum anticlockwise rotation from $\theta$.

2. The value of $s$ is decreased to $s^{\prime}$, the minimum value of the focal distance allowing the capture a sharp image of the furthest target in $Z_{\theta, s}$. More formally, $s^{\prime}$ is such that $\mathcal{D}_{\mathcal{F}}\left(s^{\prime}\right)=\max _{k \in Z_{\theta, s}}$ dist $_{i, k}+r_{t}$.

The aim of these two steps is to transform a non-normalized annular sector into a normalized one by performing a rotation of the sensing direction, then by adjusting 
Fig. 4 Original annular sector $(\theta, s)$

Fig. 5 Setting $\theta$ to $\Theta_{2}=\theta_{2}-\alpha_{2}$
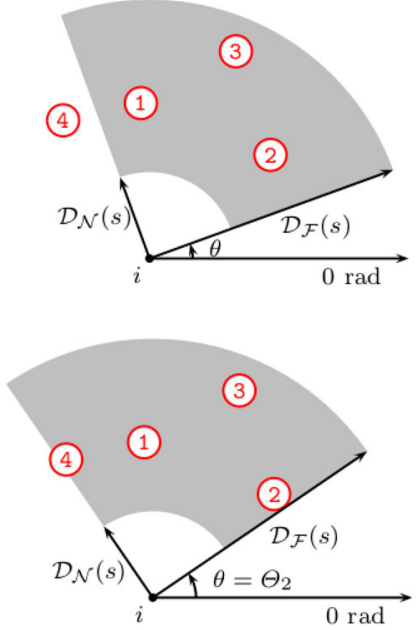

Fig. 6 Setting $s$ to $s^{\prime}$

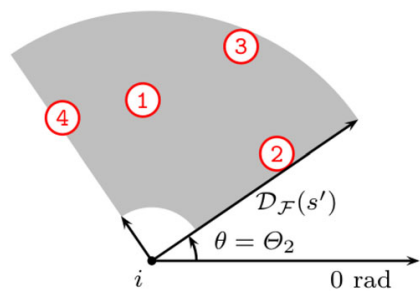

the focal distance. Doing so allows to meet the two conditions for an annual sector to be normalized. Step 1 does not compromise the coverage of the original annular sector $(\theta, s)$, as the anticlockwise rotation from $\theta$ is stopped whenever a target is found to be at the right frontier of the annular sector. Consequently, after performing the rotation, the set of covered targets is a superset of the originally covered targets, because new targets may be covered due to this rotation, whereas no target can be uncovered. Step 2 does not compromise the coverage of the original annular sector $(\theta, s)$, as the decrease of $s$ to $s^{\prime}$ is stopped whenever a target is found to be at the furthest coverage limit of the annular sector. The key hypothesis is that $\mathcal{D}_{\mathcal{N}}(s)$ and $\mathcal{D}_{\mathcal{F}}(s)$ are non-decreasing functions of $s$, which is always true with optical lenses. Finally, the lifetime of the original solution to LM-DSFD is preserved because each normalized annular sector can be used the same amount of time as the non-normalized annular sector it replaces without compromising coverage.

As an illustration of Lemma 1, Fig. 4 shows a non-normalized annual sector. The first step is shown in Fig. 5, where $\theta$ is set to $\Theta_{2}$ : we have $Z_{\theta, s} \subseteq Z_{\Theta_{2}, s}$ as target 2 limits the increase of $\theta$. When taking this step, it may happen that even more targets may be covered. However, in the case of Fig. 5, target 4 is not covered as it is not fully visible from $i$ with direction $\theta$. Finally, the second step is shown in Fig. 6, where $Z_{\theta, s} \subseteq Z_{\Theta_{k}, s^{\prime}}$, as $s^{\prime}$ is set by target 3 .

Introducing normalized annular sectors allows for reducing the number of annular sectors to at most $m \times m$ for each sensor. A sensor may cover at most $m$ targets, and 
$s$ may be adjusted to at most $m$ different values for each direction. If two normalized annular sectors cover the same set of targets, then one of them can be discarded as it can be replaced by its counterpart. Any normalized annular sector which set of covered targets is a subset of another annular sector can be discarded. This idea is implemented by the notion of dominated annular sector.

For all sensors, an annular sector $(\theta, s)$ is said to be dominated if there exists a normalized annular sector $\left(\theta^{\prime}, s^{\prime}\right)$ such that $Z_{\theta, s}$ is a strict subset of $Z_{\theta^{\prime}, s^{\prime}}$.

Lemma 2 Any solution to LM-DSFD can be transformed into a solution where nondominated annular sectors only are used, with the same lifetime.

Without loss of generality ( $c f$. Lemma 1 ), we assume that the solution to LM-DSFD relies on normalized annular sectors only. If such an annular sector $(\theta, s)$ is dominated, then it can be replaced by a normalized annular sector $\left(\theta^{\prime}, s^{\prime}\right)$ where $Z_{\theta, s}$ is a strict subset of $Z_{\theta^{\prime}, s^{\prime}}$.

\subsection{Enumeration of all the non-dominated annular sectors}

The enumeration of all non-dominated annular sectors of a given sensor is divided into two steps. First, normalized annular sectors are enumerated, then dominated ones are discarded by comparing all the couples of normalized annular sectors found so far. In the first step, not all normalized annular sectors will be enumerated: those that are obviously dominated will be immediately discarded.

\subsubsection{Step 1: enumeration of normalized annular sectors}

First, we compute $Z_{\Theta_{k}}$ as the set of all non-occluded targets that can be seen from sensor $i$ with direction $\Theta_{k}$. Next, we consider focal distance: the targets of $Z_{\Theta_{k}}$ are sorted by decreasing distance to sensor $i$, which requires $\mathcal{O}(m \log (m))$ operations. The targets in ordered set $Z_{\Theta_{k}}$ are then processed iteratively as follows.

Let $k_{1}$ be the first target in $Z_{\Theta_{k}}$. We set $s$ to $s_{k_{1}}$, the minimum value for covering $k_{1}$. By using the analytical expression of $\mathcal{D}_{\mathcal{F}}(s)$, we have $s_{k_{1}}=\frac{H\left(d i s t_{i, k_{1}}+r_{t}\right)}{H-f+d i s t_{i, k_{1}}+r_{t}}$. This defines the normalized annular sector $\left(\Theta_{k}, s_{k_{1}}\right)$ that we store, along with $Z_{\Theta_{k}, s_{k_{1}}} \subset Z_{\theta}$ as the set of all the targets that are covered by annular sector $\left(\Theta_{k}, s_{k_{1}}\right)$. Next, we consider the second target in $Z_{\Theta_{k}}$ (second in terms of distance from $i$, say target $k_{2}$ ) and we update $s$ accordingly to $s_{k_{2}}$. Note that $s_{k_{2}} \leq s_{k_{1}}$, as $\mathcal{D}_{\mathcal{F}}(s)$ is a non-decreasing function. In addition, target $k_{1}$ is now out of $Z_{\Theta_{k}, s_{k_{2}}}$ if $s_{k_{2}}<s_{k_{1}}$, whereas new targets that were not covered by annular sector $\left(\Theta_{k}, s_{k_{2}}\right)$ may now be covered by $\left(\Theta_{k}, s_{k_{2}}\right)$. So if $\left|Z_{\Theta_{k}, s_{k_{2}}}\right|<\left|Z_{\Theta_{k}, s_{k_{1}}}\right|$, then decreasing $s$ from $s_{k_{1}}$ to $s_{k_{2}}$ has just lead to compromise the coverage of $k_{1}$ without covering any new target, hence annular sector $\left(\Theta_{k}, s_{k_{2}}\right)$ is dominated. In that case, there is no need to store annular sector $\left(\Theta_{k}, s_{k_{2}}\right)$. However, it should be stressed that some of the stored annular sectors may also be dominated. The purpose of the comparison of all the couples of stored annular sectors is to discard any dominated annular sector. We then consider the third target of $Z_{\Theta_{k}}$ in terms of distance from $i$ and we repeat the process up to the last target of $Z_{\Theta_{k}}$. The enumeration 
Fig. 7 A normalized annular sector $(\theta, s)$ where $\theta$ is set by target 3

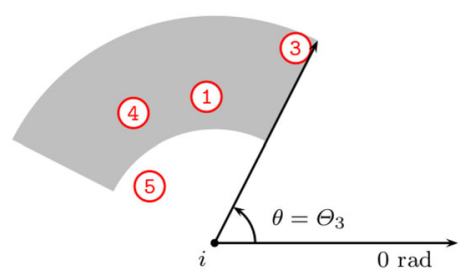

Fig. 8 A non-normalized annular sector $\left(\theta, s^{\prime}\right)$ obtained after decreasing $s$ (target 3 is no longer covered)

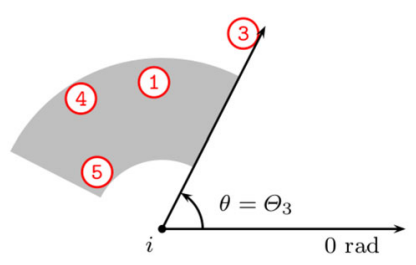

of normalized annular sectors using sensor $i$ with direction $\Theta_{k}$ is stopped if one of the following three conditions is satisfied:

- The last target of $Z_{\Theta_{k}}$ has been used for building a candidate annular sector.

- The last target in $Z_{\Theta_{k}}$ (i.e., the closest target from sensor $i$ ) is sharp with the current value of $s$. In that case, there is no new target to be covered sharply by decreasing $s$ any further, as doing so would only lead to generate dominated annular sectors. This condition can be checked by comparing the distance of this last target, denoted by $d i s t_{i, k_{\text {last }}}-r_{t}$ to $\mathcal{D}_{\mathcal{N}}(s)$. Formally, there is no need to generate additional annular sectors for sensor $i$ in direction $\Theta_{k}$ if the following inequality holds:

$$
\operatorname{dist}_{i, k_{\text {last }}}-r_{t} \geq \mathcal{D}_{\mathcal{N}}(s)
$$

Such a situation is illustrated in Fig. 6, where $k=2$, with $Z_{\Theta_{2}}=\{1,2,3\}$, and $k_{1}=3$. Decreasing $s$ from $s_{3}$ to $s_{1}$ will produce a dominated annular sector since the closest target from $i$ (target 2) is already sharply covered by annular sector $\left(\Theta_{2}, s_{3}\right)$. This means that only one annular sector is stored for sensor $i$ using direction $\Theta_{2}$ in that example.

- If the distance from $i$ to the target selected in $Z_{\Theta_{k}}$ is strictly less than dist $_{i, k}$, then target $k$, that defines direction $\Theta_{k}$, is no longer covered by any of the subsequent annular sectors to be considered, which means that these annular sectors are not normalized. Indeed, the first condition in the definition of a normalized annular sector is no longer satisfied. Hence in that case, there is no additional annular sector for sensor $i$ in direction $\Theta_{k}$ to be found. Such a situation is illustrated in Figs. 7 and 8 . In Fig. 7, target $k=3$ is used for defining direction $\Theta_{3}$, and a normalized annular sector is produced by setting $s$ to the minimum value so as to cover target 3. In Fig. 8, the direction is unchanged but after decreasing $s$ to $s^{\prime}$ which is the minimum value for covering target 4 , target 3 is no longer covered. As a result, annular sector $\left(\Theta_{3}, s^{\prime}\right)$ is not normalized. Then there is no use in decreasing $s$ any further, and a single normalized annular sector can be built from direction $\Theta_{3}$. 
For each sensor, there are $\mathcal{O}\left(\mathrm{m}^{2}\right)$ normalized annular sectors (at most $m$ different directions $\theta$, and at most $m$ different values for $s)$. Each annular sector $(\theta, s)$ is associated with $Z_{\theta, s}$, a list of at most $m$ targets covered by the considered sensor when used upon the considered annular sector.

\subsubsection{Step 2: refinement for keeping non-dominated annular sectors only}

For each sensor, let $\eta \leq m^{2}$ be the number of normalized annular sectors. For all $\left(h, h^{\prime}\right) \in\{1, \ldots, \eta\}^{2}$ such that $h<h^{\prime}$, we compute $Z_{h, h^{\prime}}$ as the union of $Z_{\theta^{h}, s^{h}}$ and $Z_{\theta^{h^{\prime}}, s^{h^{\prime}}}$, where $\theta^{h}$ is the direction of sensor $i$ associated with the $h$ th normalized annular sector, and $s^{h}$ is the corresponding focal distance.

- If $\left|Z_{h, h^{\prime}}\right|=\left|Z_{\theta^{h}, s^{h}}\right|=\left|Z_{\theta^{h^{\prime}}, s^{h^{\prime}}}\right|$, then these annular sectors are identical, and one of them (say $h^{\prime}$ ) can be discarded

- If $\left|Z_{h, h^{\prime}}\right|=\left|Z_{\theta^{h}, s^{h}}\right|>\left|Z_{\theta^{h^{\prime}}, s^{h^{\prime}}}\right|$, then $Z_{\theta^{h^{\prime}}, s^{h^{\prime}}}$ is a strict subset of $Z_{\theta^{h}, s^{h}}$, and annular sector $h^{\prime}$ can be discarded as it is dominated

- If $\left|Z_{h, h^{\prime}}\right|=\left|Z_{\theta^{h^{\prime}}, s^{h^{\prime}}}\right|>\left|Z_{\theta^{h}, s^{h}}\right|$, then $Z_{\theta^{h}, s^{h}}$ is a strict subset of $Z_{\theta^{h^{\prime}}, s^{h^{\prime}}}$, and annular sector $h$ can be discarded as it is dominated

- If $\left|Z_{h, h^{\prime}}\right|>\left|Z_{\theta^{h^{\prime}},{ }^{h^{\prime}}}\right|$ and $\left|Z_{h, h^{\prime}}\right|>\left|Z_{\theta^{h}, s^{h}}\right|$, there is no domination relation between annular sectors $h$ and $h^{\prime}$.

At the end of the process, only non-dominated annular sectors remain as no set of covered targets is a subset of any other one.

Building all non-dominated annular sectors in the sensors network can be achieved in polynomial time as it requires $\mathcal{O}\left(\mathrm{nm}^{3}\right)$ operations. Indeed, there are $n$ sensors, each of them has at most $m$ normalized directions. Each direction requires to sort at most $m$ targets by decreasing distance to the current sensor [this can be achieved in $m \log (m)$ operations] and to consider $m$ candidate normalized direction. Hence the first step requires $\mathcal{O}\left(n m^{2} \log (m)\right)$ operations.

In the second step, $n$ sensors are to be processed and for each of them, there are at most $\frac{1}{2} m(m-1)$ couples of annular sectors for which the union should be performed [in $\mathcal{O}(m)]$. Hence, the second step requires $\mathcal{O}\left(\mathrm{nm}^{3}\right)$ operations.

\section{Column generation algorithm}

The motivation for resorting to column generation is the following. Although the problem could be stated as an integer linear program (ILP), its linear programming relaxation provides a poor lower bound, and it cannot be solved efficiently with commercial solvers. Such attempts are presented in detail in Rossi et al. (2012a, b).

Let $\sigma_{i}$ be the number of non-dominated annular sectors of sensor $i$, and $g_{i}$ be defined by $g_{i}=\sum_{\ell=1}^{i-1} \sigma_{\ell}$, with $g_{1}=0$. Then, the $q$ th non-dominated annular sector of sensor $i$ has index $g_{i}+q$ for all $q \in\left\{1, \ldots, \sigma_{i}\right\}$ so all the non-dominated annular sectors for all sensors have a unique global index in $\left\{1, \ldots, g_{n}+\sigma_{n}\right\}$.

We introduce two equivalent encodings for representing a group. In the binary encoding, group $j$ is represented by a $g_{n}+\sigma_{n}$ binary vector $x$, where $x_{g_{i}+q, j}=1$ 
Table 1 From the integer vector encoding to the binary encoding

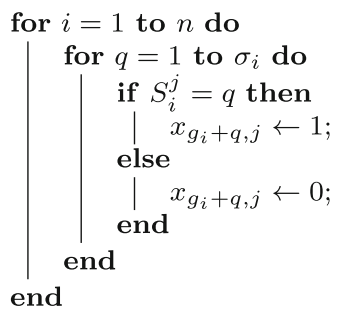

if and only if sensor $i$ is active in group $j$, and works under its $q$ th annular sector. This encoding is used in all the linear programming based formulations. In the integer encoding, group $j$ is represented by a $n$ integer vector $S^{j}$ where $S_{i}^{j} \in\left\{1, \ldots, \sigma_{i}\right\}$ is the local index of the annular sector under which sensor $i$ is used if it is active, $S_{i}^{j}$ is zero otherwise. This encoding is exploited by the GA.

Both encoding are equivalent. The integer encoding of a group can be expressed as a function of its binary counterpart as $S_{i}^{j}=\sum_{q=1}^{\sigma_{i}} q x_{i, j}$. Conversely, the algorithm in Table 1 returns the binary encoding of a group knowing its integer encoding. This algorithm is based on the following idea: if sensor $i$ works under its $q$ th non-dominated annular sector in group $j$, then $x_{g_{i}+q, j}$ is set to one, otherwise $x_{g_{i}+q, j}$ is set to zero.

As an example, let us consider a wireless sensor network made of two sensors. If the first one (respectively the second one) has three (respectively two) non-dominated annual sectors, then $\sigma_{1}=3$ and $\sigma_{2}=2$. A group where the second non-dominated annual sector of the first sensor is used has the following binary encoding: [ $\left.\begin{array}{lllll}0 & 1 & 0 & 0 & 0\end{array}\right]$, its integer encoding is [2 0]. If the integer encoding of a group is [3 1], this means that the first (respectively the second) sensor works under its third (respectively its first) non-dominated annual sector. The corresponding binary encoding is $\left[\begin{array}{lllll}0 & 0 & 1 & 1 & 0\end{array}\right]$.

The notion of dominance, that has been introduced for annular sectors can also be applied to groups. A group is said to be dominated if it contains an active sensor that can be set inactive without compromising coverage. Equivalently, if a non-dominated annular sector does not cover at least one target uniquely, then it is useless and can be removed. For any given group $j$, the algorithm shown in Table 2 computes $\Omega$, the set of all the non-dominated annual sectors of that group that cover at least one target uniquely, in the for loop. For each target $k, C_{k}$ is the set of all the non-dominated annular sectors that cover it. Then, all the non-dominated annular sectors of group $j$ that are not in $\Omega$ are removed in the forall loop, which yield a non-dominated group.

Since $\sigma_{i}$ can be up to $m^{2}$, the computational complexity of the algorithm shown in Table 1 is $\mathcal{O}\left(\mathrm{nm}^{2}\right)$, and as $g_{n}+\sigma_{n}$ can be up to $\mathrm{nm}^{2}$, the computational complexity of the algorithm shown in Table 2 is $\mathcal{O}\left(\mathrm{nm}^{3}\right)$.

Finally, any solution to LM-DSFD can be transformed into a solution using only non-dominated groups for a nonzero amount of time, these groups being uniquely constituted of sensors using non-dominated annular sectors. 
Table 2 Refining group $j$ into a non-dominated group

$\Omega \leftarrow \emptyset$;

for $k=1$ to $m$ do

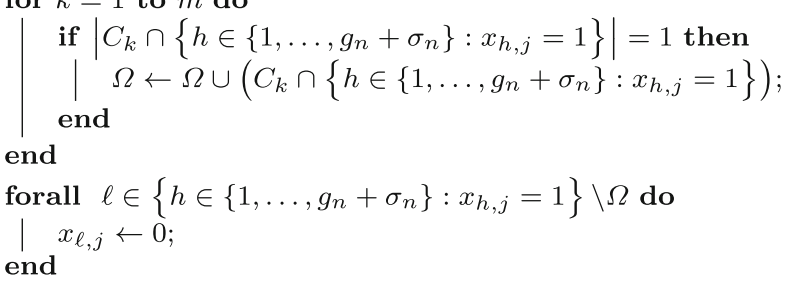

\subsection{Master problem}

The master problem is to decide the utilization time of the groups. It has $\bar{p}$ decision variables denoted by $t_{j}$ for all $j \in\{1, \ldots, \bar{p}\}$, where $\bar{p}$ is the number of groups. The master problem's constraints ensure that sensor $i$ is not used more than $b_{i}$ units of time [constraint (2)]. The dual variable associated with constraint $i$ is denoted by $y_{i}$.

$$
\begin{aligned}
& \operatorname{Maximize} \sum_{j=1}^{\bar{p}} t_{j} \\
& \sum_{j=1}^{\bar{p}}\left(\sum_{q=1}^{\sigma_{i}} x_{g_{i}+q, j}\right) t_{j} \leq b_{i} \quad \forall i \in\{1, \ldots, n\} \\
& t_{j} \geq 0 \quad \forall j \in\{1, \ldots, \bar{p}\}
\end{aligned}
$$

Since the number of groups usually grows exponentially with the number of sensors and targets, $\bar{p}$ is prohibitively large and building all the groups is impracticable even for modest size instances. We then introduce the restricted master problem, that is identical to the master problem but with a restricted number of groups $p$, that is significantly less than $\bar{p}$. From now on, $p$ refers to the number of columns of the restricted master problem at the current iteration of the column generation algorithm. Initially,

Unlike many classical problems where column generation algorithms are used, determining if LM-DSFD is feasible is a $\mathcal{N} \mathcal{P}$-complete decision problem (Cai et al. 2009). As a consequence, it is generally very difficult to initialize the restricted master problem with feasible columns, and the pricing subproblem has to be solved first, for the sake of generating a feasible group, or proving that the problem is infeasible. In order to implement this in a classical column generation framework, we initialize the master problem with a dummy column defined by $x_{i, 1}=0$ or all $i \in\left\{1, \ldots, g_{n}+\sigma_{n}\right\}$ (it is clearly not feasible) with the special constraint $t_{1}=0$ for preventing unboundedness. Doing so immediately leads to a solution with a zero lifetime, and the pricing subproblem is addressed for generating the first feasible column if there exist one.

Typical cases where the problem is infeasible include the existence of a target that is not covered by any sensor, and the existence of a unique sensor for covering two targets but with a different direction or a different focal distance. The last case is shown 
in Fig. 2 where targets 3 and 4 cannot be covered simultaneously by sensor $i$, so the problem is infeasible if that sensor only is available for covering the four targets.

\subsection{Pricing subproblem}

\subsubsection{Integer linear programming formulation}

The pricing subproblem aims at generating a group to be introduced in the restricted master problem as a new column, with index $p+1$. Group $S_{p+1}$ must have potential for allowing the network lifetime to increase. Its potential is measured by its reduced cost in the master problem. So the pricing subproblem objective function, $z=1-$ $\sum_{i=1}^{n}\left(\sum_{q=1}^{\sigma_{i}} x_{g_{i}+q, p+1}\right) y_{i}$ is the reduced cost of group $S_{p+1}$ and the constraints ensure that the solution of the pricing subproblem represents a valid group. More precisely, each sensor should be used under at most one annular sector [constraint (5)], and each target should be covered by at least one active sensor [constraint (6)]. The pricing subproblem is shown below:

$$
\begin{aligned}
& \text { Maximize } z=1-\sum_{i=1}^{n}\left(\sum_{q=1}^{\sigma_{i}} x_{g_{i}+q, p+1}\right) y_{i} \\
& \sum_{q=1}^{\sigma_{i}} x_{g_{i}+q, p+1} \leq 1 \quad \forall i \in\{1, \ldots, n\} \\
& \sum_{h \in C_{k}} x_{h, p+1} \geq 1 \quad \forall k \in\{1, \ldots, m\} \\
& x_{g_{i}+q, p+1} \in\{0,1\} \quad \forall i \in\{1, \ldots, n\}, \forall q \in\left\{1, \ldots, \sigma_{i}\right\}
\end{aligned}
$$

The number of columns $p$ in the restricted master problem is incremented by one, every time the pricing subproblem generates a group with a strictly positive reduced cost. The associated column is added to the restricted master problem that is then resolved by taking advantage of the optimal basic solution of the previous iteration, which remains feasible with the introduction of a new column.

Dominated groups may be returned by solving the pricing subproblem (i.e., useless sensors may be active), and such a situation is detrimental to convergence as new columns will have to be generated for replacing a group that contains useless sensors. Hence the algorithm shown in Table 2 is executed as a post-optimization procedure to transform a group into a non-dominated one.

\subsubsection{Heuristic solution approach}

The master problem formulation for LM-DSFD (Sect. 4.1) is a linear program. Hence, any exact solver can solve it efficiently. However, the pricing subproblem is formulated as an integer linear program (Sect. 4.2.1), and solving it can be very time consuming for any exact solver. As the column generation process requires repeatedly solving the 
master problem and the pricing subproblem alternately, it is desirable that the pricing subproblem is also solved efficiently. Therefore, a GA is also developed for addressing the pricing subproblem, and used as follows. The GA is applied first to solve the pricing subproblem. If it is able to find an attractive group (i.e., a group for which the objective value of the pricing subproblem is strictly positive), then up to $G_{\max }$ best attractive groups are returned to the master problem. The ILP formulation is used for solving the pricing subproblem only when the GA fails to find an attractive group. In case solving the ILP formulation also fails to find an attractive group, then this means that there is none, thereby proving the optimality of the current restricted master problem solution for the master problem. If solving the ILP formulation yields an attractive group, then this indicates that the GA got trapped in a local optima. Hence, the ILP formulation is used for two purposes only-either to prove the optimality of the current master problem solution, or to escape from local optima. The ILP formulation is addressed infrequently as the GA finds attractive groups most of the times. By contrast, the GA is not only several times faster, but it also returns more than just one attractive group (column) to the master problem, thereby facilitating faster convergence of the column generation process. Therefore, the use of GA expedites the entire column generation process without sacrificing the optimality of the final solution obtained through the column generation process. In this section, the term objective function refers to the pricing subproblem's objective function.

Chromosome representation Each chromosome represents a group, i.e., a potential solution to the pricing subproblem under the integer encoding scheme. There are two reasons for choosing an integer encoding scheme for our GA in place of the binary encoding scheme exploited in the master problem. First, this encoding scheme simplifies the design of crossover and mutation operators as a sensor can never have more than one direction in this encoding. Second, the integer encoding scheme yields a shorter chromosome of length $n$ in comparison to the chromosome of length $\sum_{i=1}^{n} \sigma_{i}$ with the binary encoding scheme used in the master problem. A longer chromosome length can adversely affect the execution time of a GA.

Fitness A two-level policy determines the fitness of a group (chromosome). A group is considered to be more fit than the other, if its objective function value is greater than the other. If two groups have the same objective function values, the one with a lesser number of sensors is considered to be more fit than the other. The reason behind this policy is that many groups have the same objective function value.

Selection The probabilistic binary tournament selection method has been chosen to select the two parents for crossover. In order to select a parent, two chromosomes are picked uniformly at random from the current population and their fitnesses are compared. With probability $\rho_{f}$, the chromosome having the best fitness is selected to be a parent, otherwise the chromosome having the worse fitness is selected. The second parent is selected the same way.

Crossover operator A uniform crossover operator has been used to generate a child chromosome from the two selected parents. In a uniform crossover, the value at each position of the child chromosome is copied from the corresponding position of one of the two parents. For each position, the parent whose value will be copied is chosen uniformly at random from the two parents. 
Mutation operator The mutation operator is applied to the child chromosome obtained through crossover. The value at each position of the child chromosome is checked oneby-one by the mutation operator to determine whether it is zero or non-zero. If the value at a position, say position $i$, is non-zero, then with probability $\rho_{m}$, the mutation operator resets the value at this position to zero. If the value at position $i$ is zero, then, with probability $\rho_{m}$, the mutation operator generates a random value between zero and $\sigma_{i}$ and resets the value at this position to the generated random value. Here, it is pertinent to mention that replacing a non-zero value at position $i$ with a zero corresponds to removing the sensor $i$ from the group. Alternatively, replacing a zero at position $i$ with some random value between one and $\sigma_{i}$ corresponds to adding the sensor $i$ with some randomly chosen direction into the group. Two different values of $\rho_{m}$ are used. With probability $\rho_{v}$, a child is mutated with a higher $\rho_{m}$ value, otherwise it is mutated with a lower $\rho_{m}$ value. As the child obtained after mutation may not be a feasible group, i.e., it may not cover all the targets, a repair operator is applied on the child to transform it into a feasible group.

Repair operator The repair operator consists of two stages. The first stage transforms the child into a feasible group, whereas the second stage transforms a feasible group into a non-dominated group. The first stage begins by computing a list of uncovered targets. Then, the targets in this list are considered one-by-one in some random order. For a target under consideration, it is determined whether one or more directions of a sensor not already in the child can cover this target. If there exists more than one such sensor, then the sensor whose addition decreases the objective function value the least, is selected and added into the child. The direction of this newly added sensor is randomly chosen from several directions that can cover the target under consideration. The list of uncovered targets is updated in the light of this newly added sensor. If there exist no such sensor then this means that the direction of a sensor already in the child needs to be changed to cover the target under consideration. Such targets are removed from the list of uncovered targets and placed in a list of targets requiring special processing. These targets are processed once all uncovered targets have been considered once, i.e., when the list of uncovered targets becomes empty.

The targets belonging to the list of targets requiring special processing are covered one-by-one in an iterative manner. During each iteration, a target, say $k$, is selected randomly from this list and a sensor $i$ that can cover $k$ is also selected randomly from the set of all sensors that can cover target $k$. Clearly, sensor $i$ belongs to the child, but with a direction that does not cover target $k$. To cover target $k$, the direction of sensor $i$ is changed to one of the directions that can cover $k$ (ties are broken arbitrarily) and the coverage information of all affected targets are updated. All targets including target $k$ belonging to the list of targets requiring special processing which are now covered by the new direction of $i$ are deleted from this list. However, due to the change in direction of $i$, some new targets may now no longer be covered. These targets are also added to the list of targets requiring special processing. As these newly uncovered targets can possibly be covered by sensors not present in the child, therefore, whenever such a target is selected from the list of targets requiring special processing, it is determined whether there exists a sensor $\ell$ that is not already present in the child and that can cover this target (ties are broken arbitrarily). If it is so, then $\ell$ is added to the child with a direction that can cover the target under consideration (ties are broken arbitrarily) and 
the information about the coverage of all the affected targets are updated. If no such sensor exists, then the target is processed as described already. The whole process is repeated as long as the list of targets requiring special processing is not empty. Once a child becomes feasible, it is transformed into a non-dominated group by the second stage, which consists in applying the algorithm shown in Table 2.

Replacement policy Our GA uses the steady-state population replacement model (Davis 1991). During each generation, a single child is produced which is included in the population in place of the worst member of the population in case it is different from all current population members.

Initial population generation Each member of the initial population is generated by following a two-stage procedure. The first stage begins by deciding about whether to choose all sensors in a purely random fashion or to choose some sensors in a greedy fashion and the remaining sensors in a purely random manner. The parameter $\rho_{\text {random }} \in[0,1]$ controls this decision. With probability $\rho_{\text {random }}$, the probability of greedy choice $\rho_{g c}$ is set to zero, otherwise it is set to a non-zero value. All targets are put in a list of uncovered targets. After this, an iterative process begins. During each iteration, an uncovered target is chosen randomly from this list and it is determined whether a sensor not yet included in the chromosome can cover this target. In case there are more than one candidate sensor, then with probability $\rho_{g c}$, a sensor from among these candidate sensors whose inclusion leads to the smallest reduction in the value of the objective function is added to the chromosome, otherwise a sensor is chosen randomly from among these candidate sensors and added to the chromosome. In case the target in question is covered by more than one direction of the newly added sensor, then one such direction is chosen randomly. If no sensor exists that can cover the target in question, then the direction of a sensor already present in the chromosome has to be changed to cover this target. In this case, a sensor which can cover the target in question and is already present in the chromosome is selected randomly and its direction is randomly set to one of the directions that can cover the target in question. After the target in question is covered, the coverage information of all affected targets are updated. The targets in question along with all previously uncovered targets which are now covered are deleted from the list of uncovered targets, and any target which becomes uncovered now is added to the list of uncovered targets and another iteration begins. This process is repeated until the list of uncovered targets becomes empty, i.e., all targets are covered. The second stage of initial population generation process is identical to the second stage of repair operator, i.e., it transforms a chromosome generated through the first stage into a non-dominated group. Each newly generated chromosome is compared with the initial population members generated so far, and if it is found to be unique, then it is included in the initial population, otherwise it is discarded. This procedure is repeated until the desired number of distinct population members are generated.

Our initial solution generation process can get stuck into an infinite loop in case no feasible group exists, i.e., when the problem instance is infeasible. In this case, our initial solution generation process will keep on changing cyclically the directions of sensors. To prevent this from happening, while generating an initial solution we keep track of the number of times the directions of sensors that are already present in the solution got changed. If the directions of the sensors got changed more than 
$D_{\max }$ times, then the initial population generation process is stopped and the GA is terminated with an indication that it failed to find a feasible cover.

Another possibility where our initial solution generation process can be trapped into an infinite loop is the case where the number of different feasible solutions is less than the size of the population. In this case, our initial solution generation process will start discarding solutions continuously. To prevent this situation, if the initial population generation process fails to find a solution which is different from current population members even after $T_{\max }$ consecutive trials, then population size is reset to the size of the current population.

Other features Our GA terminates when the fitness of the best solution does not improve over $I_{\max }$ consecutive iterations. However, the value of $I_{\max }$ is not same for all the runs of the GA. The value of $I_{\max }$ varies over the set $I_{\text {choices }}=$ $\{50,200,400,800,1200,1600\}$. For those instances with more than 50 sensors, the value of $I_{\max }$ is initialized to 200 , whereas it is initialized to 50 for those instances with lesser sensors. If the GA fails to find $G_{\max }$ attractive groups, then the value of $I_{\text {max }}$ for the next run of the GA is set to the next higher value, if there is any, in $I_{\text {choices }}$, otherwise it is set to the next lower value, if there is any, in $I_{\text {choices }}$. The value of $I_{\max }$ is never allowed to fall below its initial value in $I_{\text {choices }}, e . g$., for those instances with more than 50 sensors the value of $I_{\max }$ can never be set below 200 .

Numerical tests have shown that the use of a GA is beneficial to speed up the column generation algorithm at the early stage of the search, i.e., when numerous and various columns can be found to increase the lifetime significantly. However, column generation is known to exhibit the so-called tailing-off effect (Lübbecke and Desrosiers 2005): at the end of the search, new columns provide only a very modest amount of increase in lifetime, and a large number of columns is required to reach the optimal solution and prove its optimal status. To this respect, the focus on solution quality in addressing the pricing subproblem becomes more and more prevalent while the benefit of the diversity offered by a population based method becomes less and less advantageous. Hence, the use of an ILP solver appears to be more helpful in efficiently completing the search, and is more efficient than a GA to cope with the tailing-off effect. Consequently, we switch off the GA permanently whenever the increase in lifetime from one iteration of the column generation process to the next is less than a given threshold $L_{T}$, and the ILP formulation is solved only afterwards.

\subsection{Upper bounds on lifetime}

Sometimes, the column generation algorithm may take a prohibitively long time to converge, and because of the tailing-off effect, a very large amount of time may be required to close a very small gap to optimality. In that case, the user may want to interrupt the search and use the current solution of the master problem. We propose two upper bounds on the lifetime. The first one is static, i.e., it is computed before starting the column generation algorithm, and will not be updated during the search. The second upper bound is a dual upper bound that can be updated every time the pricing subproblem is solved to optimality. 


\subsubsection{Static upper bound}

Let $\bar{C}_{k}$ be the set of sensors that can cover target $k$, and $u_{k}$ be the maximum amount of time during which target $k$ can be covered if all of its neighboring sensors are used for that:

$$
u_{k}=\sum_{i \in \bar{C}_{k}} b_{i}
$$

Then, $\min _{k \in\{1, \ldots, m\}} u_{k}$ is an upper bound on the lifetime. This upper bound can be improved by considering all the pairs of targets $\left(k, k^{\prime}\right)$ such that there exists at least one sensor $i$ whose distance to each of them is less than $R$, and also such that none of its annual sector contains both $k$ and $k^{\prime}$. In that case, sensor $i$ has to be shared between $k$ and $k^{\prime}$, i.e., it cannot be used $b_{i}$ units of time for covering both $k$ and $k^{\prime}$.

Then, for all pairs of targets $k$ and $k^{\prime}$, we define $S_{k k^{\prime}}$ as the set of all the sensors that can cover $k$ and $k^{\prime}$, but that cannot cover them simultaneously. More formally, $i$ belongs to $S_{k k^{\prime}}$ if the three following conditions hold:

1. $C_{k} \cap\left\{g_{i}+1, \ldots, g_{i}+\sigma_{i}\right\} \neq \varnothing$

2. $C_{k^{\prime}} \cap\left\{g_{i}+1, \ldots, g_{i}+\sigma_{i}\right\} \neq \varnothing$

3. $C_{k} \cap C_{k^{\prime}} \cap\left\{g_{i}+1, \ldots, g_{i}+\sigma_{i}\right\}=\emptyset$

If $\left|u_{k^{\prime}}-u_{k}\right|<\sum_{i \in S_{k k^{\prime}}} b_{i}$, then assuming without loss of generality that $u_{k}<u_{k^{\prime}}$, the quantity $u_{k}-\frac{1}{2}\left(\sum_{i \in S_{k k^{\prime}}} b_{i}-\left(u_{k^{\prime}}-u_{k}\right)\right)$ is also a lower bound that is strictly less than $u_{k}$. This quantity is the maximum amount of time during which targets $k$ and $k^{\prime}$ can be covered, it has been computed by dispatching the $\sum_{i \in S_{k k^{\prime}}} b_{i}$ units of time between $k$ and $k^{\prime}$ so as to maximize the minimum amount of coverage among them (in this case, both $k$ and $k^{\prime}$ have the same coverage time). Consequently, the static upper bound $U B_{1}$ is defined by:

$$
U B_{1}=\min \left(\min _{k \in\{1, \ldots, m\}} u_{k}, \min _{\substack{\left(k, k^{\prime}\right) \in\{1, \ldots, m\}^{2} \\ u_{k^{\prime}}-u_{k}<\sum_{i \in S_{k k^{\prime}}} b_{i}}} u_{k}-\frac{1}{2}\left(\sum_{i \in S_{k k^{\prime}}} b_{i}-\left(u_{k^{\prime}}-u_{k}\right)\right)\right)
$$

As an illustration, let us consider two targets $k$ and $k^{\prime}$ such that $u_{k}=5, u_{k^{\prime}}=6$, and $\sum_{i \in S_{k k^{\prime}}} b_{i}=2$. Since the sensors in $S_{k k^{\prime}}$ cannot cover $k$ and $k^{\prime}$ simultaneously, the upper bound on the lifetime, that is currently set by $u_{k}=5$, can be improved to $U B_{1}=\min \left(5,6,5-\frac{1}{2}(2-1)\right)=4.5$. 


\subsubsection{Dual upper bound}

The dual upper bound on the lifetime introduced below is updated every time the pricing subproblem is solved to optimality. The dual of the master problem is:

$$
\begin{aligned}
& \operatorname{Minimize} \sum_{i=1}^{n} b_{i} y_{i} \\
& \sum_{i=1}^{n}\left(\sum_{q=1}^{\sigma_{i}} x_{g_{i}+q, j}\right) y_{i} \geq 1 \quad \forall j \in\{1, \ldots, \bar{p}\} \\
& y_{i} \geq 0 \quad \forall i \in\{1, \ldots, n\}
\end{aligned}
$$

Let $\tilde{y}$ be the optimal dual solution of the restricted master problem, and let $z^{\star}$ be the optimal objective value of the pricing subproblem. We then have $\sum_{i=1}^{n}\left(\sum_{q=1}^{\sigma_{i}} x_{g_{i}+q, j}\right) \tilde{y}_{i}+z^{\star} \geq 1$, for all group $j \in\{1, \ldots, \bar{p}\}$.

If $\eta \geq 1$ is a lower bound on the number of sensors in any feasible group, then vector $\pi$ defined by $\pi_{i}=\tilde{y}_{i}+\frac{z^{\star}}{\eta}$ can be shown to be a feasible solution to the dual of the master problem. By the weak duality theorem (Chvátal 1983), the optimal lifetime is less than $U B_{2}=\sum_{i=1}^{n} b_{i} \pi_{i}$.

For $U B_{2}$ to be as tight as possible, $\eta$ should be set to the minimum cardinality of any feasible group. This can be done by solving the pricing subproblem with $\tilde{y}_{i}=1$, and by setting $\eta=1-z^{\star}$. If the problem instance is too difficult for solving the pricing subproblem to optimality in reasonable time, then $\eta$ can be set to $1-\left\lfloor z^{L P}\right\rfloor$, where $z^{L P}$ is the optimal objective value of the linear programming relaxation of the pricing subproblem.

\section{Computational results}

A set of 180 instances is used to compare the three problem versions, they have been generated as follows. The number of sensors and targets, $(n, m)$ is in the range $\{(50,30),(100,30),(200,120),(400,240)\}$. Thus, the ratio $m / n$ is $60 \%$, as in most of the benchmarks in the literature (Wang et al. 2009b; Rossi et al. 2013). The monitoring angle $\varphi$ is in the range $\{90,120,180\}$ (in degrees), and the targets are discs of radius $r_{t}$ in the range $\{2,4,8\}$, except for the directional sensor problem, for which the targets are represented as dimensionless points. For each triplet $\left((n, m), \varphi, r_{t}\right)$, five different instances are generated randomly, making a total of $4 \times 3 \times 3 \times 5=180$ instances.

The maximum amount of time allotted to each single instance has arbitrarily been set to one hour, as in most works on wireless sensor networks in the literature. All the algorithms have been implemented in $\mathrm{C}$ and executed on a PC running Linux Ubuntu 14.04 LTS with an Intel Core i7-4790 processor at $3.6 \mathrm{GHz}$ equipped with $32 \mathrm{~GB}$ RAM. All integer and linear programs were solved using IBM CPLEX 12.6.2.

The values of different parameters of the GA are shown in Table 3. All these parameter values have been chosen empirically after a large number of trials. These 
Table 3 Values of different parameters of the GA

\begin{tabular}{|c|c|c|}
\hline Parameter & Value & Comments \\
\hline$G_{\max }$ & 25 & $\begin{array}{l}\text { Our GA can return a maximum of } 25 \text { best attractive } \\
\text { groups to the master problem. It may return lesser } \\
\text { number of attractive groups in case the total number } \\
\text { of attractive groups is less that } G_{\max }\end{array}$ \\
\hline Population size & $\min (n, 50)$ & - \\
\hline$\rho_{f}$ & 0.8 or 0.9 & $\begin{array}{l}\text { In probabilistic binary tournament selection, the first } \\
\text { parent is selected with } \rho_{f}=0.8 \text { and the second one is } \\
\text { selected with } \rho_{f}=0.9\end{array}$ \\
\hline$\rho_{v}$ & 0.1 & Governs the value of mutation probability $\rho_{m}$ \\
\hline$\rho_{m}$ & 0.02 or 0.01 & $\begin{array}{l}\text { With probability } \rho_{v}, \rho_{m} \text { is set to } 0.02 \text {, otherwise it is set } \\
\text { to } 0.01\end{array}$ \\
\hline$\rho_{\text {random }}$ & 0.66 & $\begin{array}{l}\text { Governs the value of greedy selection probability } \rho_{g c} \\
\text { while generating each member of initial population }\end{array}$ \\
\hline$\rho_{g c}$ & 0 or 0.4 & $\begin{array}{l}\text { With probability } \rho_{\text {random }}, \rho_{g c} \text { is set to } 0 \text {, otherwise it is } \\
\text { set to } 0.4\end{array}$ \\
\hline$D_{\max }$ & $2 \times m$ & $\begin{array}{l}\text { Initial population generation process is terminated if no } \\
\text { feasible group can be found even after changing } \\
D_{\max } \text { directions }\end{array}$ \\
\hline$L_{T}$ & $5 \times 10^{-5}$ & $\begin{array}{l}\text { Whenever increase in lifetime from one iteration of } \\
\text { column generation process to the next is less than } L_{T} \text {, } \\
\text { then we switch off the GA permanently }\end{array}$ \\
\hline$T_{\max }$ & 10 & $\begin{array}{l}\text { During initial population generation process, the GA } \\
\text { resets the population size to the current population } \\
\text { size if it fails to find a new population member } \\
\text { different from current population members even after } \\
T_{\max } \text { consecutive trials, and, during the generations of } \\
\text { the GA, the GA terminates if it fails to generate a } \\
\text { child solution different from current population } \\
\text { members even after } T_{\max } \text { consecutive trials }\end{array}$ \\
\hline
\end{tabular}

values provided good results on all the instances, though they may not be optimal for any instance.

The results for the three problem versions considered in this paper are displayed in Table 4. Problem ' $d$ ' is the same as in Rossi et al. (2013): directional sensors are considered, with dimensionless targets. Problem ' $\mathrm{g}$ ' (' $\mathrm{g}$ ' stands for geometry) is an extension of ' $d$ ', where targets dimensions and occlusion are taken into account. Finally, Problem 'f' ('f' stands for focal) is an extension of ' $g$ ', where focal distance is considered. Each row in the table represents a set of five randomly generated instances. The instance names are built as follows: n050m030a090t02 means that $n=50$ sensors, $m=30$ targets, the sensor monitoring angle $\varphi$ is $90^{\circ}$ and the targets are discs of radius $r_{t}=2$. The sensing range of the sensors is set to $R=150$ in all the instances, and all sensors have an initial energy allowing them to be active for one unit of time.

For each problem version, LT is the average lifetime achieved, UB is the average best upper bound on the lifetime [i.e., it is the average of $\left.\min \left(U B_{1}, U B_{2}\right)\right]$, and CPU is the average computational time in seconds spent for finding an optimal solution. If 


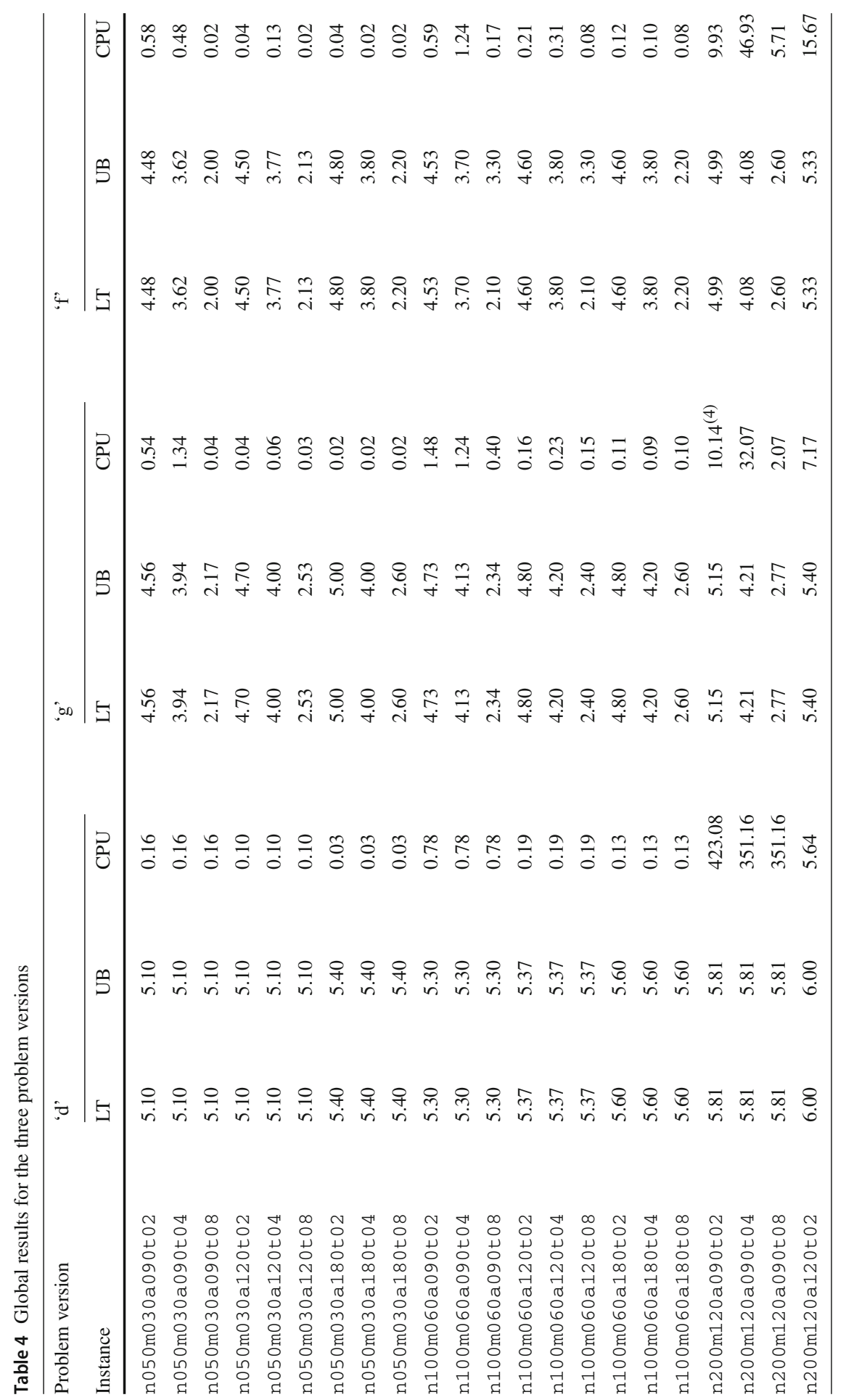


Table 5 Detailed results of problem 'g' on the set n200m120r150a090t02

\begin{tabular}{llll}
\hline Instance number & LT & UB & CPU \\
\hline 0 & 6 & 6 & 10.56 \\
1 & 4.33333 & 4.33333 & 0.66 \\
2 & 6.61602 & 6.61751 & 3600.00 \\
3 & 5.77778 & 5.77778 & 28.97 \\
4 & 3 & 3 & 0.36 \\
\hline
\end{tabular}

one or more instances among the five ones is not solved to optimality because the time limit was reached, then a superscript indicates in parenthesis the number of optimal solutions found, over which the CPU time is computed.

Since the directional problem version ' $d$ ' ignores the targets size, the results are exactly the same for all the instances that only differ by the radius. These instances are grouped by three (their name ends with t 02 , t 04 and t 08 in Table 4). However, it can be observed that this is not always the case for the average CPU time of instance sets n200m120a090t02 and n200m120a090t04 for example. This is because the CPU time is computed only on the maximum set of instances that have been solved to optimality for the three problem versions together. The superscript (4) on the CPU column of problem version ' $d$ ' with $r_{t}=2$ in Table 4 indicates that the average CPU time has been computed on four instances, because ' $\mathrm{g}$ ' has been solved to optimality for four instances only. For $r_{t}=4$, the average CPU time for ' $\mathrm{d}$ ' is different because it has been computed on five instances. This is due to the fact that ' $d$ ', ' $g$ ' and 'f' have been solved to optimality for all the instances of the set n200m120a090 t 04 . A slightly different situation arises with instance sets n400m240a090t02 and n400m240a090 t04. In both cases, only four instances out of five were solved to optimality for ' $\mathrm{d}$ ', but the four-instance set is not the same in both cases, which leads to different results.

The last row of Table 4 displays the average lifetime, upper bound and computational time for the three problem versions. The wireless sensor network lifetime decreases from ' $d$ ' to ' $g$ ' and to ' $\mathrm{f}$ ' as the problem versions are enriched with more constraints. The lifetime decrease is much more significant when the radius of targets is large. Indeed, in that case, occlusion is much more likely to happen. By contrast, the lifetime drop due to focal distance is less.

When all the instances are not solved to optimality, the gap to the upper bound is very small. Table 5 shows the detailed results for the five instances of the set $\mathrm{n} 200 \mathrm{~m} 120 \mathrm{a} 090 \mathrm{t} 02$ for problem version ' $\mathrm{g}$ '. Instance number 2 is the only one that is not solved to optimality in this set, with LT $=6.61602$ and $\mathrm{UB}=6.61751$. The gap is too small to be visible in the averaged results of the table (we read 5.15 for the average lifetime and for the upper bound as well in Table 4). The reason why the gap is so small is that the dual upper bound is very tight at the end of the search. Actually, after one hour of computation, the column generation algorithm is subject to the tailing-off effect when solving this particular instance. These small gaps suggest that the proposed approach can serve as a good quality heuristic, and the availability of the dual upper bound may allow the user to stop the search prematurely if she or 
he is satisfied enough with the current solution quality, knowing that only marginal improvements can be expected, possibly at a large computational cost.

As expected, the results shown in Table 4 indicate that the computational difficulty of the three problem versions is increasing with the problem size. But it seems that the monitoring angle $\varphi$ has an even more significant impact on computational time. Instances with a small monitoring angle are much more difficult than those with a larger angle. The problem of deciding if ' $\mathrm{d}$ ' is feasible is $\mathcal{N} \mathcal{P}$-complete (Rossi et al. 2013), but it is polynomial for omni-directional sensors. Consequently, the practical difficulty of building feasible groups increases when the monitoring angle $\varphi$ decreases.

\section{Conclusions}

This work could be complemented and extended in at least two directions. First, one could consider that a base station gathers all the data harvested by the sensors, and that the sensors are too far away to communicate directly with the base station. In that case, a communication network based on multi-hop communication should be considered. The second research direction would be to enrich the model with even more physical parameters. For example, a three-dimensional model could be considered, by introducing an azimuth angle to each camera, and providing three-dimensional coordinates for targets and sensors. Another interesting parameter to consider is the ability for a camera to zoom in and out. Doing so would not only have an impact on sharpness, but it would also change the monitoring angle. Both these modifications could be done without impacting the structure of the column generation algorithm. The pricing subproblem might become more difficult as more parameters should be set at each iteration, and the preliminary step of building non-dominated annual sectors might be more complex and time consuming than in the present study.

\section{References}

Astorino, A., Gaudioso, M., Miglionico, G.: Lagrangian relaxation for the directional sensor coverage problem with continuous orientation. Omega 75, 77-86 (2018)

Cai, Y., Lou, W., Li, M., Li, X.-Y.: Energy efficient target-oriented scheduling in directional sensor networks. IEEE Trans. Comput. 58, 1259-1274 (2009)

Chvátal, V.: Linear Programming. W.H Freeman and Company, New York (1983)

Davis, L.: Handbook of Genetic Algorithms. Van Nostrand Reinhold, New York (1991)

De, D., Gupta, M., Sen, A.: Energy efficient target tracking mechanism using rotational camera sensor in WMSN. In: Proceedings of the Second International Conference on Communication, Computing \& Security, Procedia Technology, Vol. 6, pp. 674-681 (2012)

Garcia-Sanchez, A., Garcia-Sanchez, F., Garcia-Haro, J.: Wireless sensor network deployment for integrating video-surveillance and data-monitoring in precision agriculture over distributed crops. Comput. Electron. Agric. 75(2), 288-303 (2011)

Guo, L., Li, D., Zhu, Y., Kim, D., Chen, W.: Enhancing barrier coverage with $\beta$ quality of monitoring in wireless camera sensor networks. Ad hoc Netw. 51, 62-79 (2016)

Guvensan, M., Yavuz, A.: On coverage issues in directional sensor networks: a survey. Ad hoc Netw. 9(7), 1238-1255 (2011)

Guvensan, M., Yavuz, A.: Hybrid movement strategy in self-orienting directional sensor networks. Ad hoc Netw. 11(3), 1075-1090 (2013) 
Han, L., Zhou, M., Jia, W., Dalil, Z., Xu, X.: Intrusion detection model of wireless sensor networks based on game theory and an autoregressive model. Inf. Sci. 476, 491-504 (2019)

Hong, Y., Yan, R., Zhu, Y., Li, D., Chen, W.: Finding best and worst-case coverage paths in camera sensor networks for complex regions. Ad hoc Netw. 56(1), 202-213 (2017)

Ji, Y., Yamashita, A., Asama, H.: Automatic calibration of camera sensor networks based on 3D texture map information. Robot. Auton. Syst. 87, 313-328 (2017)

Jianjian, D., Yang, T., Feiyue, Y.: A novel intrusion detection system based on IABRBFSVM for wireless sensor networks. Proc. Comput. Sci. 131, 1113-1121 (2018)

Li, X., Yan, L., Pan, W., Luo, B.: Optimization of DV-hop localization algorithm in hybrid optical wireless sensor networks. J. Heuristics 21(2), 177-195 (2015)

Li, F., Luo, J., Xin, S., He, Y.: Autonomous deployment of wireless sensor networks for optimal coverage with directional sensing model. Comput. Netw. 108, 120-132 (2016)

Lin, Y., Saluja, K., Megerian, S.: Adaptive cost efficient deployment strategy for homogeneous wireless camera sensors. Ad hoc Netw. 9(5), 713-726 (2011)

Liu, X.: Chapter 106 A Survey on Wireless Camera Sensor Networks in Frontier and Future Development of Information Technology in Medicine and Education, Lecture Notes in Electrical Engineering, Vol. 269, pp. 1085-1094 (2014)

Lübbecke, M.E., Desrosiers, J.: Selected topics in column generation. Oper. Res. 53(6), 1007-1023 (2005)

Queiroz, D., Alencar, M., Gomes, R., Fonseca, I., Benavente-Peces, C.: Survey and systematic mapping of industrial wireless sensor networks. J. Netw. Comput. Appl. 97, 96-125 (2017)

Rossi, A., Singh, A., Sevaux, M.: A column generation algorithm for sensor coverage scheduling under bandwidth constraints. Networks 60(3), 141-154 (2012a)

Rossi, A., Singh, A., Sevaux, M.: An exact approach for maximizing the lifetime of sensor networks with adjustable sensing ranges. Comput. Oper. Res. 39, 3166-3176 (2012b)

Rossi, A., Singh, A., Sevaux, M.: Lifetime maximization in wireless directional sensor network. Eur. J. Oper. Res. 231, 229-241 (2013)

Sharmin, S., Narin Nura, F., Razzaque, M., Rahman, M., Alelaiwi, A., Hassan, M., Rahman, S.: $\alpha-$ Overlapping area coverage for clustered directional sensor networks. Comput. Commun. 109, 89-103 (2017)

Sung, T.-W., Yang, C.-S.: Voronoi-based coverage improvement approach for wireless directional sensor networks. J. Netw. Comput. Appl. 39, 202-213 (2014)

Tao, D., Tang, S., Zhang, H., Mao, X., Ma, H.: Strong barrier coverage in directional sensor networks. Comput. Commun. 35(8), 895-905 (2012)

Wang, J., Niu, C., Shen, R.: Priority-based target coverage in directional sensor networks using a genetic algorithm. Comput. Math. Appl. 57(11-12), 1915-1922 (2009a)

Wang, C., Thai, M.T., Li, Y., Wang, F., Wu, W.: Optimization scheme for sensor coverage scheduling with bandwidth constraints. Optim. Lett. 3, 63-75 (2009b)

Wang, T., Zeng, J., Bhuiyan, M.D., Chen, Y., Cai, Y., Tian, H., Xie, M.: Energy-efficient relay tracking with multiple mobile camera sensors. Comput. Netw. 133, 130-140 (2018)

Yang, C., Zhu, W., Liu, J., Chen, L., Chen, D., Cao, J.: Self-orienting the cameras for maximizing the view-coverage ratio in camera sensor networks. Pervasive Mob. Comput. 17(Part A), 102-121 (2015)

Yick, J., Mukherjee, B., Ghosal, D.: Wireless sensor network survey. Comput. Netw. 52(12), 2292-2330 (2008)

Publisher's Note Springer Nature remains neutral with regard to jurisdictional claims in published maps and institutional affiliations. 